Historic, Archive Document

Do not assume content reflects current scientific knowledge, policies, or practices. 



\section{J A N U A R Y 1915}

凪

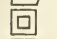

回

맘

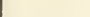

\section{TRADE}

LIST

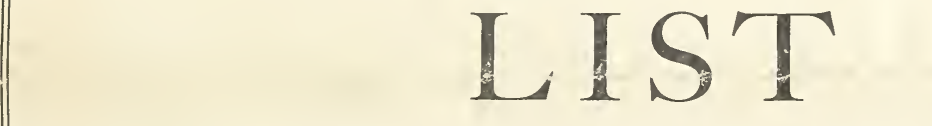

每 NEWARK, Wayne County, NEW YORK 


\section{Terms and Conditions of Sale}

This list cancels all previous quotations.

PRICES. The prices named in this list are based upon present market conditions, and are subject to change without notice. We invite correspondence with prospective buyers, and shall be pleased to quote special prices on want lists calling for larger quantities than are named herein. On the other hand, these are wholesale prices and are applicable only to wholesale quantities; in no case will we fill retail orders, even though from nurserymen, at these prices. All orders must be made up in 10's or multiples of 10 . Otherwise we reserve the right to decline them or to fill the orders at prices consistent with the quantity ordered.

PACKING. Will be done with every care, and always with the destination of the shipment in mind; we send Roses, Perennials, and other stock to the extreme South and to Pacific Coast points and rery rarely have any loss in transit; owing to superior packing.

BOXES AND BALES will be charged for at actual cost of materials used. We make no charge for packing or for delivery to the railroad stations. We invite comparison of our charges with what others ask for the same sized boxes. Ours cost:

\begin{tabular}{|c|c|}
\hline 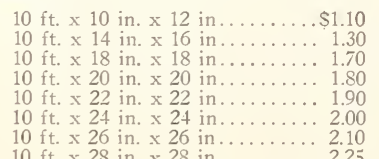 & 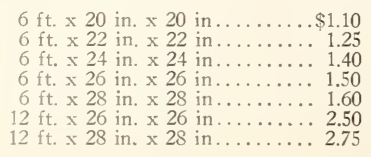 \\
\hline
\end{tabular}

SHIPPING. We have the New York Central, the West Shore, and the Pennsylfaxia Rarlroads, and the Americax, Adans, National and Wells Fargo Express, enabling us to ship in any direction orer trunk lines. All shipments travel at the risk and cost of the purchaser.

TERMS. Usual terms to those of established credit or whose responsibility is linow'n to us. Cash, security, or satisfactory references required of unknown parties.

All agreements and contracts are made subject to crop losses through unavoidable causes.

GUARANTY. Wc use crery precaution to have all our stock true to label, and should any prove not to be so, we will replace it without charge or refund the purchase price, but all orders are accepted br us upon the mutual agrcement between purchaser and oursclves, that we shall not be responsible for other damages.

CLAIMS. For any cause, must be made promptly on receipt of goods. Any stock that is not exactly as represented, mav be returned to us immediately on arrival, and we will cheerfully and witlout question. credit back the unsatisfactory plants, or send others; but we will not entertain claims made after goods have been once accepted, or when report is not made within six dars after arrival of the stock.

INSPECTION AND FUMIGATION. Certificate will be furnished with every shipment, and when required stock will be fumigated.

SPECIAL NOTICE. Correspondents are requested to use their printed business letter-heads. We do not quote prices to rctail buvers nor sell to planters; we have no retail trade of our own, and sell to the trade only; in justice to our only customers, the Nurservmen. Florists. and large dealers, who do their own packing, we use every effort to limit the distribution of trade prices to those we know to be entitled to receive them. Use printed stationery.

LOCATION. We are located thirty miles east of Rochester, N. Y., and cordially invite a visit and inspection of our establishment.

$$
\text { Respectfully, }
$$

JACKSON \& PERKINS COMPANY.

Newark, New York, January 23, 1915. 


\section{$R O S E S$ Field Grown, Two Years}

R O S E - G R O W I N G has been our specialty ever since we began the nurscry business. The accompanying pictures, from photographs taken in our blocks last summer, and the following extract from an article in "The Florists Exchange," of September 5, 1911, will convey some idea of the extent of our propagation of hardy field-grown Roses:

"When Mr. Perkins informed the writer that their annual output of Roses was more than a million and a half, it was evident there were some great treats in store for a visitor. Although too late to sce them in all their glory, there were sufficient to give one an idea of what the sight must have been. All the best old varieties and the really good new ones are grown. The demand for Baby Ramblers is steadily increasing, and this year there are over 75,000 ; the sight they present, one is not likcly to forget, and the writcr was much impressed with the correctness of the stock, for block after block, one could not detect a wrong color. These, like all the Roses, have made a splendid growth, the scason having been very favorable. Frau Karl Druschki, Gruss an Teplitz, White and Pink Killarney, and several other kinds had some remarkably good flowers."

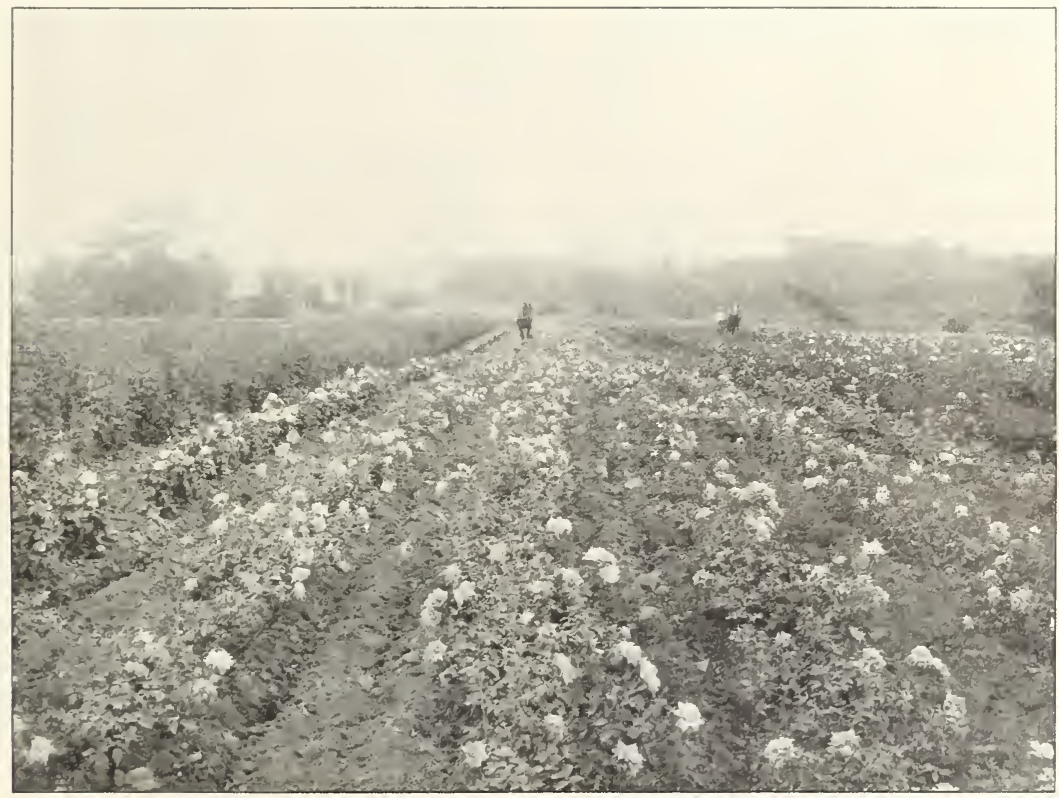

A 1914 Block of Hybrid Tea Roses

On own-roots, except where marked (b), which indicates budded plants.

GRADES-No. 1 are our best and largest plants.

Having no retail trade of our own, sending out neither catalogs, nor agents, and selling nothing to planters, our ONI.Y customers - the Nurserymen and Florists - can expect the best we can grow.

No. $1 \frac{1}{2}$ or Medium Grade consists of plants just under first-class, such as do not have the neeessary number of branches, or are slightly under weight and height. They will be carefully selected, healthy plants, with good roots and tops, only slightly lighter than first grade.

No. 2 Grade. These plants we especially recommend to Nurserymen for transplanting, and with ordinary good care and cultivation they should make fine, strong stock in a single season. Many of our customers consider them good cuough for immediate sale. They are carefully selected, evenly-graded plants, very suitable for mailing or for usc where an extra heavy grade is not required.

We cannot undertake to supply long and much assorted lists of stock calling for only small quantities of each kind, save at an advance of 10 to 20 per cent. above our printed rates. 


\section{RAMBLERS and CLIMBERS

American Pillar, pink, single $385 @ 10 \mathrm{c}$ Anna Maria, blush..........35@7c

Baltimore Belle, blush....... 300@8c

Birdie Bly (b) carmine.......1 $130 @ 10 \mathrm{c}$

Cherokee (b) white......... $36 @ 10 c$

Climbing American Beauty,

red ...................1000@25c

Climbing American Beauty, Climbing Baby Rambler

(Miss G. Messman) red...555@10c Climbing Mrs. Cutbush, pink $315 @ 10 c$

Crimson Rambler ..........2200@10c

Crimson Rambler (b)........3000@10c

Dorothy Perkins ..........6000@8c

Dorothy Perkins (b).........350@8c

Excelsa (Red Dorothy

Perkins).................1000@10c

Flower of Fairfield (Ever-

blooming Crimson

Rambler) ...............5 $505 @ 12 c$ (b) ...................440@25c

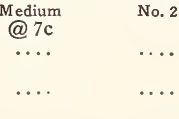

380
No. 1

Flower of Fairfield (b)......1900@12c Goldfinch (Paul's) yellow...1000@8c Hiawatha, crimson, single...1000@10c Lady Gay, pink..............1000@8c Lady Godiva (b) flesh pink.. $65 @ 8 c$ Mme. Ghys, mauve..........180@8c Miss G. Messman (Climbing Oriole, yellow .............1100@8c Philadelphia, crimson ......1500@10c Queen of the Prairies, red...45@7c Tausendschon (b).........185@10c Tennessee Belle, rosy blush.365@8c

Trier, yellow cream........425@8c Veilchenblau (Blue Ram-

bler) violet blue...........140@8c White Dorothy Perkins.....1000@8c Wichuriana (type) ...........3700@8c Yellow Rambler ............1000@8c
Baby Rambler) ...........565@10c Tausendschon, pink ........4600@10c

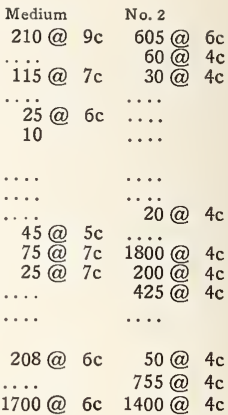

Blanda, pink with red fruit.. 60 Lucida, bright pink with

orange-scarlet fruit ...... 30

Nitida, low, bushy with pink

flower .................. 110

30

\section{MOSS ROSES}

Rubrifolia, pink with scarlet

No.1 1 Medium

@ 7c

No. 2

fruit ................ 84

Setigera, rose .......... 90

10

$\cdots$

No. 2

No. 5

$50 @ 5$

192

$\begin{array}{lr}\ldots . & 35 \\ \cdots & 73\end{array}$

is

145

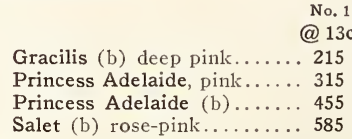

No. 1

@ $13 \mathrm{c}$

Medium

@ 7c

....

.

30

No. 2

(a) $5 \mathrm{c}$

55

35

20

\section{DWARF POLYANTHA or BABY ROSES}

Except as noted

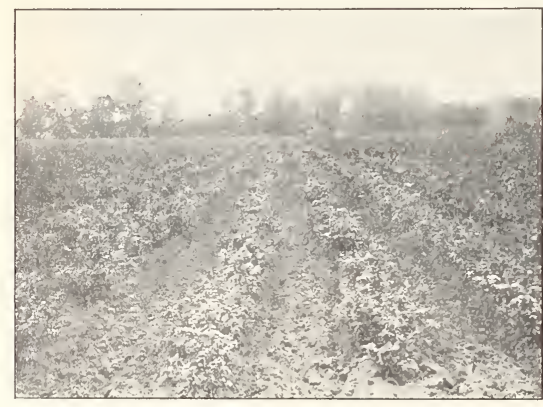

Baby Rambler. J. \& P. 1914 Block.

\section{@ $15 \mathrm{c}$}

Anny Muller, pink............

Baby Dorothy, pink...........

Baby Dorothy (b) $\ldots \ldots \ldots \ldots \ldots$

Baby "Rambler ............ 25

Baby Rambler (b)..........6 6000

Baby Tausendschon, pink... 340

Baby Tausendschon (b) ......1350

Ellen Poulson (b) pink ..... 90

Erna Teschendorff (b) car-

mine - red............. 560

Jessie (scarlet Baby Rambler) 680

Jessie (b) ..............1615

Mrs. Cutbush, pink.............4 400

Mrs. Cutbush (b) ......... 500

Orleans, red ............... 75

Orleans (b) .............. 240

Rodhatte (b) cherry-red.... 40

White Baby Rambler........... 1675

White Baby Rambler (b) .... 70

Yellow Baby Rambler.......29 @20c

Yellow Baby Rambler (b)...300@20c

$$
\begin{array}{r}
80 \\
140 \\
60
\end{array}
$$

Mediu

@ 10c

No. 2

140
60

90
2110

2110
535

....

140
700

700
560

245

400

125

37

950

50

$75 @ 15 c$

$255 @ 15 c$
@ $6 \mathrm{c}$

25

200

25

85

1750

1090

....

50

650

400

50

320
75

6

650

20

$180 @ 10 \mathrm{c}$

$300 @ 10 c$

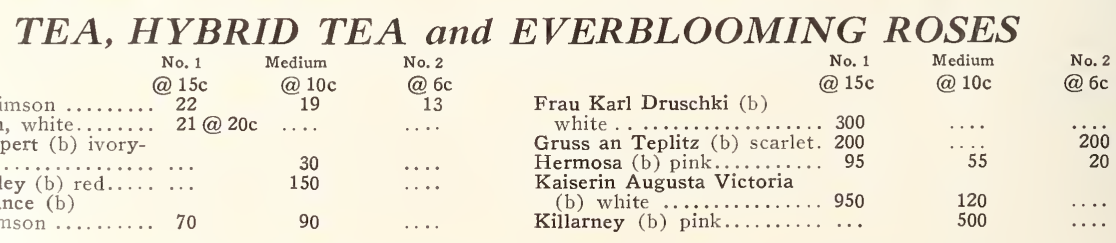

This list is intended for the trade only, and the prices given will be applied only when the quantities required are such as to entitle the purchaser to wholesale rates. 


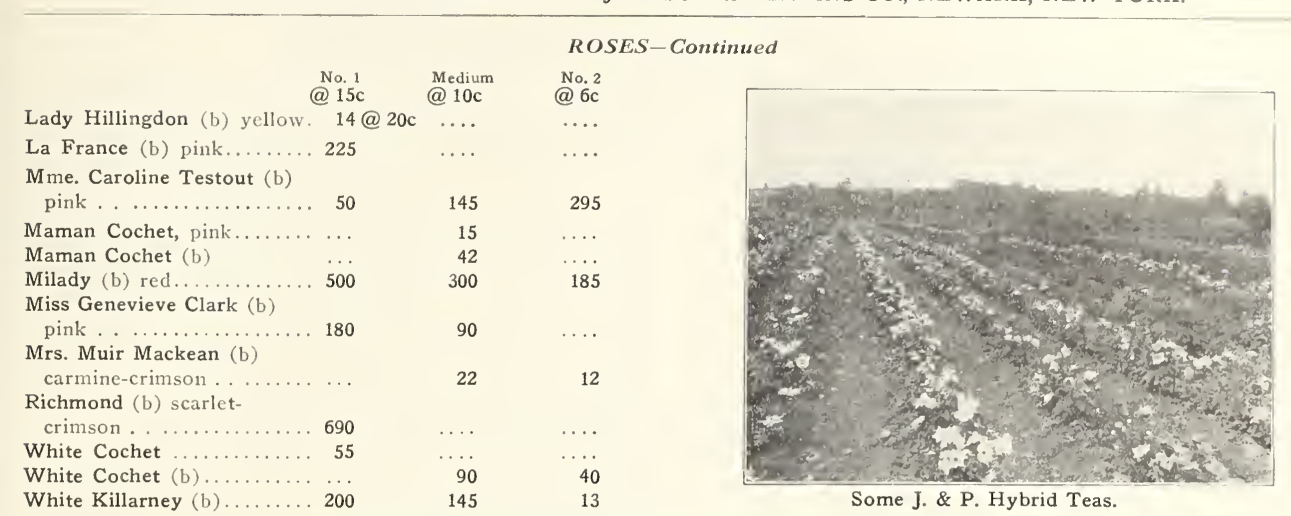

\section{HYBRID PERPETUAL AND MISCELLANEOUS ROSES}

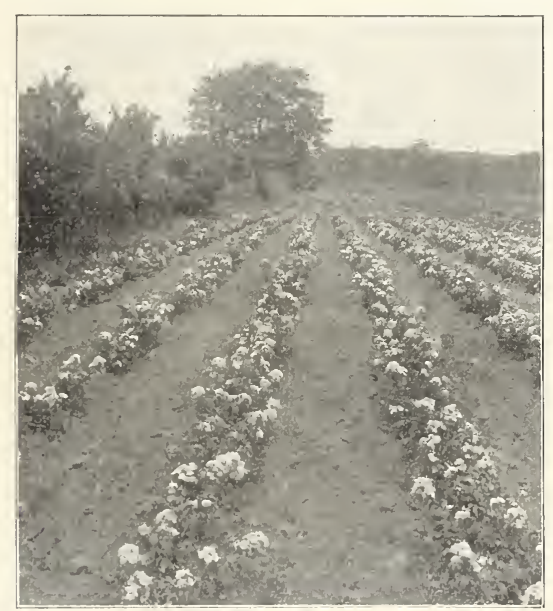

Pink Baby Rambler

\begin{tabular}{|c|c|c|}
\hline Exce & ept as noted & \\
\hline No. 1 $12 \mathrm{c}$ & $\begin{array}{l}\text { Medium } \\
@ 7 \mathrm{c}\end{array}$ & $\begin{array}{l}\text { No. } 2 \\
@ 5 \mathrm{c}\end{array}$ \\
\hline Alfred Colomb (b) crimson. 400 & & \\
\hline American Beauty (b) red....2000@15c & $800 @ 10 c$ & $2350 @ 6 c$ \\
\hline Anne de Diesbache (b) pink. 2500 & $\ldots .$. & 570 \\
\hline Baron de Bonstettin (b) & & \\
\hline maroon . . . . . . . . . 1750 & $\cdots$ & 660 \\
\hline Black Prince (b) very dark.. 165 & $\ldots$. & 10 \\
\hline $\begin{array}{l}\text { Baroness Rothschild (b) } \\
\text { light pink } \ldots . \ldots \ldots \ldots 215\end{array}$ & & \\
\hline light pink ................. & 125 & 140 \\
\hline $\begin{array}{l}\text { Captain Hayward (b) } \\
\text { bright scarlet ........... }\end{array}$ & & \\
\hline $\begin{array}{l}\text { bright scarlet .............. } \\
\text { Caroline Marniesse, white, }\end{array}$ & $\cdots \cdot$ & $\cdots \cdots$ \\
\hline $\begin{array}{c}\text { Caroline Marniesse, White, } \\
\text { everblooming . . . . . } 180 . . .\end{array}$ & 125 & 90 \\
\hline
\end{tabular}

OHIO, December 2, 1914.- "Roses this year are better than any I have ever reecived. I am more than pleased with the stoek."

\begin{tabular}{|c|c|c|}
\hline @o.1 & $\begin{array}{l}\text { Medium } \\
@ 7 \mathrm{c}\end{array}$ & No. 2 \\
\hline Clio (b) flesh colored........ 885 & 60 & 65 \\
\hline Coquette des Alps (b) white. 30 & .... & 50 \\
\hline Coquette des Blanches, white ... & & 65 \\
\hline $\begin{array}{l}\text { Coquette des Blanches (b).. } 395 \\
\text { Eugene Furst (b) dark }\end{array}$ & $\cdots$ & 35 \\
\hline crimson . . .......... 790 & $\ldots$. & 150 \\
\hline $\begin{array}{l}\text { Fisher Holmes (b) crimson.. } 540 \\
\text { Gloire Lyonnaise (b) }\end{array}$ & $\ldots$. & 100 \\
\hline $\begin{array}{c}\text { creamy white } \ldots \ldots \ldots \ldots \\
\text { General Jacqueminot (b) }\end{array}$ & 34 & 80 \\
\hline crimson ..............3800 & & \\
\hline General Washington, crimson 175 & 45 & 220 \\
\hline General Washington (b) ... 200 & & 65 \\
\hline $\begin{array}{l}\text { Harrison's Yellow (b) ....... } 315 \\
\text { Hugh Dickson crimson. }\end{array}$ & 45 & 80 \\
\hline Hugh Dickson (b) ......... 500 & 25 & $\begin{array}{l}85 \\
70\end{array}$ \\
\hline John Keynes (b) maroon.... 50 & & 59 \\
\hline John Hopper, rose......... 10 & 17 & 40 \\
\hline John Hopper (b) ......... 600 & & 245 \\
\hline J. B. Clark, crimson....... 12 & 20 & 9 \\
\hline $\begin{array}{l}\text { J. B. Clark (b) } \\
\text { Mme. Gabriel Luizet, rose... . . }\end{array}$ & 18 & $\begin{array}{r}50 \\
140\end{array}$ \\
\hline Mme. Gabriel Luizet (b) ...2700 & & 100 \\
\hline Mme. Plantier, white....... 500 & & $\ldots .$. \\
\hline Magna Charta, pink........ 760 & 300 & .... \\
\hline $\begin{array}{l}\text { Magna Charta (b) ............ } 4000 \\
\text { Marchioness of Dufferin, }\end{array}$ & $\cdots$ & $\cdots$ \\
\hline Marchioness of Lorne & 25 & 8 \\
\hline crimson ............... 16 & & 40 \\
\hline Marchioness of Lorne (b)... 10 & 14 & 10 \\
\hline $\begin{array}{l}\text { Margaret Dickson (b) white. ... } \\
\text { Marie Bauman (b) crimson- }\end{array}$ & $\cdots$ & 165 \\
\hline $\begin{array}{l}\text { vermilion } \\
\text { Marshall P. Wilder (b) }\end{array}$ & .... & 50 \\
\hline scarlet-crimson ........ 445 & & 300 \\
\hline $\begin{array}{l}\text { Mrs. John Laing (b) pink... . . } \\
\text { Mrs. R. G. S. Crawford (b) }\end{array}$ & 140 & 730 \\
\hline 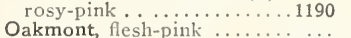 & 43 & 260 \\
\hline Paul Neyron (b) pink........ 1300 & .... & \\
\hline Persian Yellow (b) $\ldots \ldots \ldots \ldots 1950$ & $\ldots$ & 450 \\
\hline $\begin{array}{l}\text { Prince Camille de Rohan (b) } \\
\text { crimson } \ldots \ldots \ldots \ldots \ldots \ldots \ldots 1340\end{array}$ & & \\
\hline Tom Wood (b) cherry-red....... & 30 & 13 \\
\hline $\begin{array}{l}\text { Ulich Brunner (b) crimson. } 2000 \\
\text { Victor Verdier, carmine-red. ... }\end{array}$ & 140 & $\begin{array}{r}550 \\
55\end{array}$ \\
\hline
\end{tabular}

IXDIANA. Norember 16, $191 \%$ - "The Roses have proven more than satisfaetory-I have never seen finer Roses than these."

We cannot undertake to supply long and much assorted lists of stock calling for only small quantities of each kind, save at an advance of 10 to 20 per cent. above our printed rates. 


\title{
RUGOSA and HYBRID RUGOSA ROSES
}

This we consider our most valuable class of Roses wherever hardiness is a requisite to success in rose-growing. Combining the vigor and strength of the Rugosa-its luxurient and persistent foliage,-with size, fragrance, quality and everblooming habit with no loss of hardiness, the resulting hybrids possess nearly all the requisites of the ideal rose for cold climates.

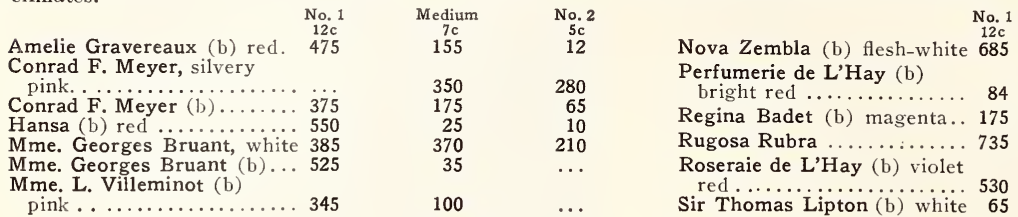

$\begin{array}{rr}\begin{array}{c}\text { Medium } \\ 7 \mathrm{c} \\ 145\end{array} & \begin{array}{c}\text { No. } 2 \\ 5 \mathrm{c}\end{array} \\ & 50 \\ 48 & \\ 50 & 105 \\ 120 & 65 \\ & \\ 114 & 60 \\ 55 & 10\end{array}$

\section{STANDARD or TREE ROSES}

\$20 the Hundred; Small Lots, 30c Each

9 Alister Stella Gray, lemon yellow.

30 Apotheker Georg Hofer, carmine.

13 Archiduchesse Miarie Immaculata, rosy-yellow

53 Augustine Guinoisseau, white La France.

3 Baby Rambler.

5 Barbarosa, red.

40 Betty, coppery rose and golden yellow.

21 Clothilde Soupert, blush.

15 Coquette Des Blanches, white.

3 Crimson Rambler.

14 Dean Hole, salmonish rose.

60 Deutchland, golden yellow.

29 Duchess of Wellington, deep yellow and orange.

7 Farbenkonigin, brilliant rose.

24 Freiherr von Marschall, carmine.

20 Franciska Kruger, flesh color.

48 Frau Karl Druschki, white.

10 General Jacqueminot, crimson.

7 General Mac Arthur, red.

14 Gloire de Dijon, coppery yellow and salmon.

11 Gruss an Teplitz, scarlet.

20 Grace Darling, yellowish-rose.

7 Gustav Grunerwald, yellowish-rose.

7 Her Majesty, rose.

17 J. J. L. Mock, rose-carmine.

25 Jules Margottin, pink.

10 Kaiserin Augusta Victoria, white.

16 Killarney, pink.

7 Koningin Emma, white, rosy shaded.
$19 \mathrm{La}$ Detroit, tender rosy and white.

26 Lady Ashtown, pure rose.

19 Lady Hillingdon, yellow.

15 La France, pink.

77 L'Hollande, white.

44 La Reine, blush.

60 Lyon, salmon and yellow.

$28 \mathrm{La}$ Tosca, tender rosy with white and yellow.

68 Mme. Abel Chatenay, yellowish-rose.

70 Mme. Caroline Testout, pink.

19 Mme. Edmee Metz, light rosy.

19 Mme. Jeanne Dupuy, reddish yellow.

30 Mme. Jules Grolez, red.

69 Mme. Leon Pain, rosy with some orange.

24 Mme. Ravary, orange yellow.

6 Magna Charta, pink.

6 Marechal Neil, yellow climber.

10 Mildred Grant, ivory.

15 Mrs. Aaron Ward, deep yellow.

5 Mrs. Joseph Hill, salmon.

6 Mrs. John Laing, pink.

7 Paul Neyron, pink.

17 Pharisaer, light rose.

5 Prince de Bulgarie, yellowish rose, center orange.

28 Souvenir de Pierre Notting, golden.

47 Souvenir de Mme. Helene Lambert, chamois.

11 Viscountess Folkestone, blush.

63 White Killarney.

\section{$C L E M A T I S$}

Our success in growing Clematis free from the dreaded disease found in the Holland-grown plants, and the demand for the home-grown stock, has enabled us to hold the lead as the largest growers of Clematis in the world.

We grade our Clematis in three sizes, as we do our Roses, and offer two grades under No. 1, which are described as follows:

No. $1 \frac{1}{2}$ grade will consist of plants just under first grade. They will be good thrifty stock, splendid plants for mail orders and suitable anywhere that an extra heavy grade is not required.

No. 2 grade will also do very nicely for mail trade, being healthy, well-rooted plants. We also recommend them for transplanting and growing on, as they will make fine, strong plants very quickly.

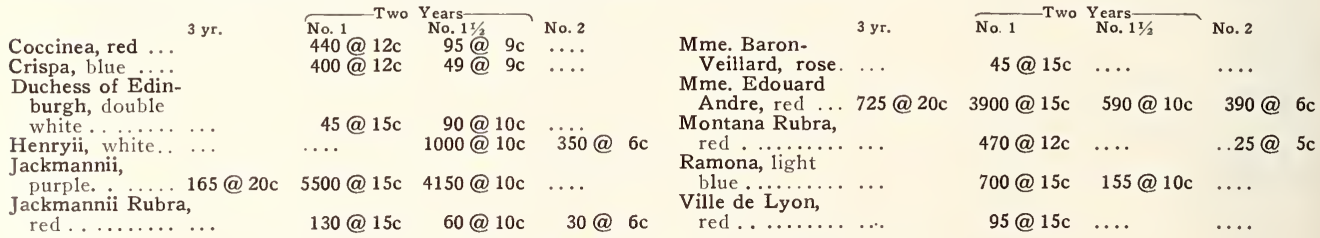

\section{CLEMATIS PANICULATA}

\author{
4000 Paniculata, 3 yrs \\ Per M $-P e r C$ \\ 5000 Paniculata, 2 yrs. No. 1 ..............\$85.00 \\ Per C
$\$ 10.00$ \\ 8.00 \\ 2000 Paniculata, 2 yrs., No. $11 / 2 \ldots \ldots \ldots \ldots \ldots \ldots . \$ 20.00$




\section{CLIMBING VINES}

Field Grown

4000 Ampelopsis Engelmanni, No, 1 Per $\mathbb{M}$

250 Ampelopsis Engelmannii, No, $11 / 2 \ldots \ldots \ldots \ldots \ldots$

1600 Ampelopsis Quinquefolia, No. $1 \ldots \ldots \ldots \ldots \ldots . . \ldots \ldots$

200 Ampelopsis Quinquefolia, No. $1 \frac{1}{2} \ldots \ldots \ldots \ldots$

4000 A mpelopsis Veitchii, $3 \mathrm{yr}, \ldots . . . \ldots . .85 .00$

5000 Ampelopsis Veitchii, 2 yr., No. 1....... 65.00

500 Ampelopsis Veitchii, 2 yr., No. $11 / 2 \ldots \ldots \ldots \ldots \ldots$

1100 Ampelopsis Veitchii, 2 yr., No. $2 \ldots \ldots \ldots \ldots \ldots$

75 Aristolochia Sipho (Dutchman's Pipc)

120 Aristolochia Sipho (Dutchman's Pipc),
Per C

$\$ 8.00$

5.00

6.00

4.00

10.00

8.00

6.00

4.00

20.00

15.00
270 Celastrus Scandens, 3-4 ft.

65 Celastrus Scandens, 2-3 ft.

125 Honeysuckle, Chinese Twining, No. 1

40 Honeysuckle, Chinese Twining, No. 11

4000 Honeysuckle, Hall's Japan, No, 1

145 Honeysuckle, Monthly Fragrant, No. 1

75 Honeysuckle, Monthly Fragrant, No. 1

13 Honeysuckle, Reticulata Aurea, No. 1 .

80 Honeysuckle, Scarlet Trumpet, No. 1

1300 Wistaria, Chinese Purple, No. 1

800 Wistaria, Chinese Purple, No. 11/2.

300 Wistaria, Chinese White, No. 1 $\begin{array}{cc}\text { Per M } & \text { Per C } \\ \$ \ldots . . & \$ 8.00\end{array}$

6.00

4.00

4.00

$60.00 \quad 7.00$

8.00

6.00

8.00

8.00

12.00

9.00

20.00

\section{HARDY PERENNIAL PLANTS}

All strong field-grown stock; all are easily grown and when once establishcd are permanent; the most satisfactory plants for the amateur's garden.

300 ANCHUSA, Italica Dropmore $\ldots \ldots \ldots \ldots \ldots \ldots \ldots \ldots \ldots$
ANEMONE JAPONICA $\ldots \ldots \ldots \ldots \ldots \ldots \ldots .50 .00$

AQUILEGIA (Columbine) $\ldots \ldots \ldots \ldots \ldots . . .45 .00$
345 California Hybrids.

300 Canadensis, red and yellow.

255 Chrysantha, yellow.

335 Chrysantha Alba, white.

265 Coerula, (Rocky Mountain Columbine)

150 Veitch's Long Spurred.

CHRYSANTHEMUMS, Hardy Pompon . 45.00

450 Autumn Beauty, golden brown.

670 Baby, lcmon yellow (miniature.)

260 Diana, pure white.

285 Fireball, ycllow tipped with crimson.

90 Garza, white.

260 Julia, orange-scarlet.

175 Julia La Graverre, rich garnet.

180 Klondike, ycllow.

180 Ladysmith, lilac, tinted salmon.

60 Mary Colloday, lavender pink.

700 Minta, light pink.

290 Nio, white.

330 Orea, light pink.

230 Queen of Whites, creamy whitc.

260 Quinola, yellow.

770 Tennyson, light pink

265 Zenobia, pure yellow.

DELPHINEUMS (Larkspur)

135 Amos Perry.

175 Amyas Leigh.

135 Chinensis Alba.

35 Chinensis Mixed.

23 Gold Medal Hybrids.

23 Mme. Violet Geslin.

135 Rembrandt.

2165 DIANTHUS BARBATUS (Sweet William) 45.00 DIANTHUS PLUMARIUS (Hardy Scotch Pinks) 360 White Reserve.

350 DIGITALIS PURPUREA (Foxglove).........

370 DIELYTRA SPECTABILIS (Bleeding

Heart)

FRAXINELLA. $\ldots \ldots \ldots \ldots \ldots \ldots \ldots \ldots \ldots \ldots$ 140 Alba.

190 Rubra.

1000 GAILLARDIA, Kelway's Grandiflora Superba

5600 GYPSOPHILLA, Paniculata Flore Plena (Double Baby's Breath) ....... 80.00

HIBISCUS . .

1300 Crimson Eye.

380 Moscheutos.

5.00

6.00
HOLLYHOCKS, doublc, strong, field- Per M PerC grown plants .............\$40.00 \$ 5.00 2000 Allegheny (singlc).

1700 Maroon.

1800 Pink.

3200 Red.

2800 Salmon.

2500 White.

2100 Yellow.

265 HYPERICUM MOSERIANUM (Gold

Flower) .............

IRIS KAEMPFERI (Japanese), named

varieties ........................... creamy standards.

150 Boteki-No-Koe, single white purple veined, lavender standards.

70 Ho-O-Miya, mauve.

55 Idzumi-Gawa, double whitc, purple veined, yellow at base of petals.

25 Karako-Asob, very double dark blue, with darker veins, ycllow at base of petals.

200 Matsu-Kaze, doublc, dark purplc yellow at base of petals.

50 Momiji-Go-Taki, crimson purplc, feathered whitc.

85 Otorige, light purple, end of pctals dark purple, claret colored standards tipped white.

325 Shishi-Ho, double bluc, veined whitc, base of petals ycllow.

45 Shishi-Ikari, crimson falls, shading to white at basc, dark ponciling, standards whitc, red tippcd, ycllow blotches.

70 Shikai-Nami, bluc.

135 Shirago, dark purple, six petals.

110 Shu-Chu-No-Hana, double white, pctals edged with red, base of pctals yellow.

140 Sumida-Gawa, single white, light bluc markings, standards shaded light bluc, ycllow at base of petals. Very large.

150 Ushio-No-Komuri, delisitc bluc, end of petals darker.

195 Yodo-No-Kuruma, single, pinkish, darker veined, yellow at basc of petals.

20 Zama-No-Mori, delicatc lavender with orance blotches.

6000 Mixed, all colors

$\$ 30.00 \$ 5.00$

We cannot undertake to supply long and much assorted lists of sto:k calling for only small quantities of each kind, save at an advance of 10 to 20 per cent. above our printed rates. 


\section{HARDY PERENNIAL PLANTS-Continued}

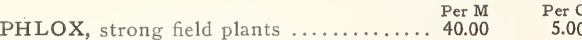

2500 Beranger, white suffused with pink; rosy-lilac eye.

4000 Bridesmaid, pure white with large crimson center.

4000 Coquette, white with pink eye.

1200 Fraulein G von Lassburg, pure white; immense panicles.

1800 Iris dark; purple violet.

2300 La Vague, pure mauve with anilinred eye.

500 Le Mahdi, deep, velvety purple, large spike.

3700 Lothair, cerise-salmon with crimson eye.

375 Lumineaux, carmine and rose with blush center.

C

4000 Mme. Bezanson, best crimson.
85 Richard Wallace, white with carmine eye.

3000 Sir Edwin Landseer, bright crim-

son.

2700 Sunset, dark, rosy-pink.

60 Thebaide, carmine-lake with bright shadings.

PAPAVER (Poppy)

\section{P E O N I ES}

Strong divisions with three to five good eyes and some roots.

300 ALBION, cherry rose, edged paler and blush-white, full double $\ldots \ldots \ldots \ldots \ldots \ldots \ldots 10.00$ 30 CAMILLE CALOT, light rose .................... 10.00

75 CHARLEMAGNE, creamy white, center light lilac,

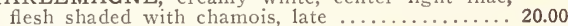

50 CLARISSA, rosy pink; inner petals sulphur......... 6.00

150 COMTE de DIESBACH, dark red, early ............ 10.00

75 COMTE de NIEPPERG, dark rose-pink; large bloom 10.00

100 COMTE d'OSMOND, white with sulphurish center.. 12.00

25 COURONNE d'OR, immense, full, imbricated, ballshaped bloom; snowy white with yellowish stamens; fragrant; perfect as a cut flower sort;

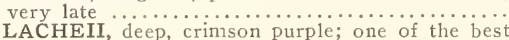

25 DELACHEII, deep, crimson purple; one of the best dark kinds, late $\ldots$ EHOURS, the CALOT variety.

60 DUCHESSE DE NEMOURS, the CALOT variety

100 DUKE OF WELLINGTON, fragrant, sulphur-white large and well formed; strong grower; stems long and firm; late

250 FESTIVA MAXIMA, early, white; fine for florist's work ATIVA ALBA, ivory-white, with occasional crimSon spots in center petals; ships well; late, mid-
son

150 FRANCOIS ORTEG̈̈̈. semi-double; large, purplishcrimson bloom with brilliant, golden yellow anthers.

50 GRANDIFLORA ROSEA, light rose; outer petals very large; inner smaller and bunched; vigorous

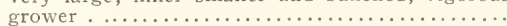

75 HESPERIA, pink

50 LAIUS, outer petals white, inner sulphur, changing to white ................................. blooms, borne on long, erect stems, delicate rose, fading to creamy-white; one of the best........ 20.00

170 LOUIS VAN HOUTTE, (Delachei), fine dark crim-

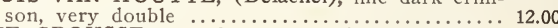

100 MME. CROUSSE, one of the best whites, midseason. 15.00

25 M'LLE. RENE DESSERT, in clusters, soft lilac with

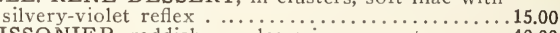

100 MEISSONIER, reddish purple; crimson center . . . . . . 40.00

50 NIGRICANS, brilliant, purplish-crimson, very late... 20.00

40 NOBILISSIMA, bright, deep pink; good stems, lasts

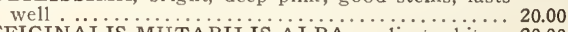

50 OFFICINÄLIS MUTABILIS ALBA, earliest white.. 20.00

75 PAPAVIFLORA, guard petals ptire white, others yellowish. 15.00

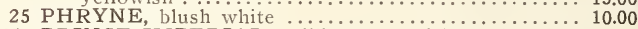

450 PRINCE IMPERIAL, brilliant, purplish-scarlet; free

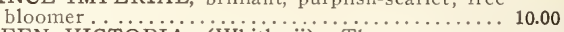

500 QUEEN VICTORIA, (Whitleyii). There are two well-known varieties of this name. This is the standard pure white market variety for storage.. 10.00

50 RICHARDSON'S DORCHESTER, salmon-pink, one

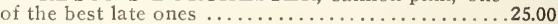

25 ROSEA PLENA SUPERBA, very full flower, delicate

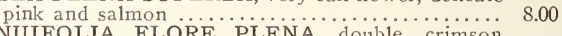
50 TENUIFOLIA FLORE PLENA, double crimson, deeply cut, fern-like foliage ............... 25.00 100 TOROUEMADA, a fine peach-colored variety ......... 10.00 125 JAPAN TREE. PEONIES, all colors, mixed....... 40.00

\section{CONIFERS}

Thrifty, shapely, well-furnished plants, twiee-transplante d, anyway.

ARBOR VITAE

20 American, 4-5 ft.

500 American, 3-4 ft.

2000 American, 2-3 ft.

380 American, $18-24$ in.

200 American, $12-18 \mathrm{in}$.

15 Hoveyi, 18-24 in.

55
740 Pyramidal, $2-3 \mathrm{ft}$...

740 Pyramidal, $18-24$ in.
630 Pyramidal, $12-18$ in.

150 Siberian, $12-18$ in.

JUNIPER

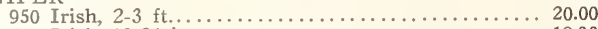

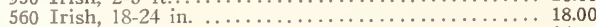

PINE

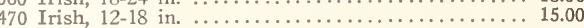

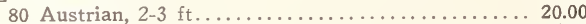

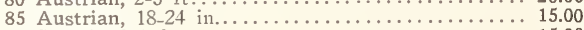

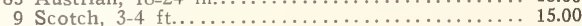

45 Scotch, $18-24$ in. .............................. 10.00

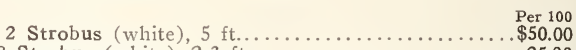

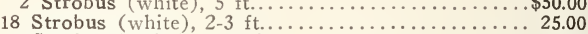

45 Strobus (white), $18-24$ in ...................... 20.00

SPRUCE

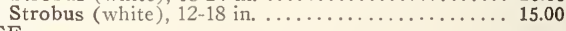

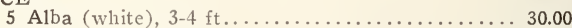

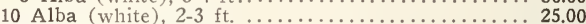

30 Alba (white), 18-24 in. 20.00

95 Colorado, selected blue, $2-3 \mathrm{ft} . \ldots \ldots \ldots \ldots \ldots \ldots \ldots . \ldots \ldots . \ldots \ldots$

80 Colorado, selected blue, $18-24$ in. ........................... 750

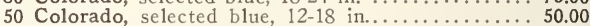

10 Colorado, green specimens, $18-24$ in ......... 15.00

175 Hemlock, $18-24$ in. ................................. 200

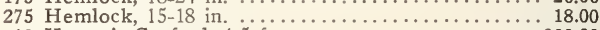

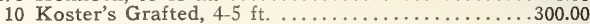

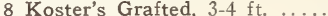

900 Norway, 3-4 ft. ... . . . . . . . . (Per 1000 . \$120) . 15.00

2400 Norway, $2-3 \mathrm{ft} . \ldots \ldots \ldots \ldots \ldots$. Per $1000 \ldots$... 85) .. 10.00

3600 Norway, $12-18$ in. ..................... (Per 1000.. 50$)$.. 6.00

This list is intended for the trade only, and the prices given will be applied only when the quantities required are such as to entitle the purchaser to wholesale rates. 


\section{HARDY DECIDUOUS SHRUBS}

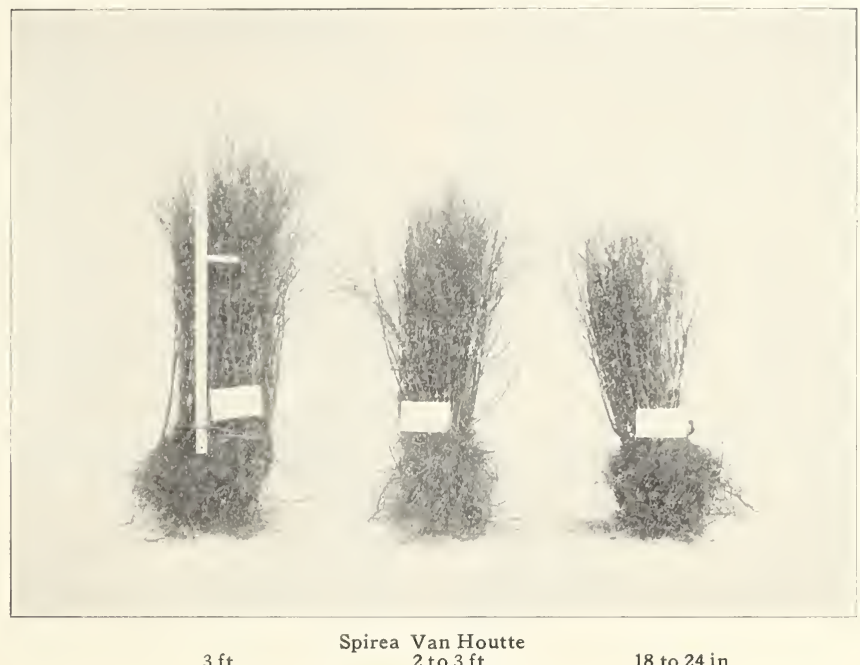

$3 \mathrm{ft}$.

$$
2 \text { to } 3 \mathrm{ft} \text {. }
$$

18 to 24 in

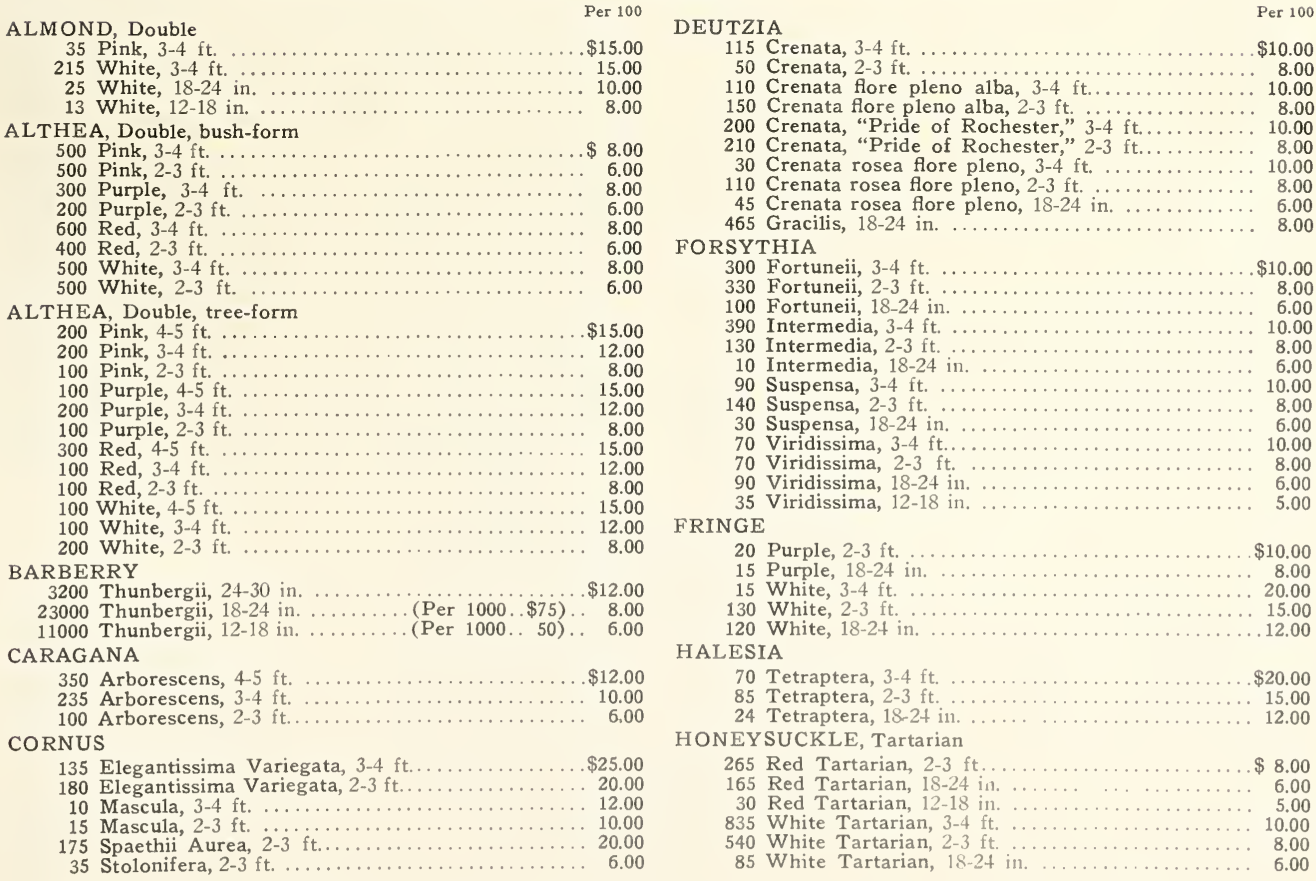

We cannot undertake to supply long and much assorted lists of stock calling for only small quantities of each kind, save at an advance of 10 to 20 per cent. above our printed rates. 


\section{HARDY DECIDUOUS SHRUBS-Continued}

Per 100

HYDRANGEA

RIBES

350 Arborescens Sterilis (Grandiflora Alba) 12-18 in. $\$ 10.00$ For Hydrangea Otaksa and other forcing varieties, see page 15 .

\section{Hydrangea Paniculata Grandiflora}

3500 Standard or Tree-form, $4-5 \mathrm{ft} . \ldots \ldots \ldots \ldots \ldots \ldots . \ldots 30.00$

700 Standard or Tree-form, $3 \mathrm{~T} / 2-4 \mathrm{ft} . \ldots \ldots \ldots \ldots \ldots \ldots \ldots \ldots . \ldots . .25 .00$

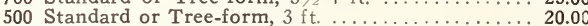

100 Standard or Tree-form, 2-3 ft. . . . . . . . . . . 15.00

2100 Bush-form, 2-3 ft. . ..................... 10.00

845 Bush-form, 18-24 in. . . . . . . . .

JAPAN QUINCE

1100 Japan Quince, $2-3 \mathrm{ft} . \ldots \ldots \ldots \ldots \ldots \ldots \ldots \ldots . \ldots 8.00$

830 Japan Quince, $18-24$ in. ...................... 6.00

KERRIA

225 Japan Quince, 12-18 in. . . . . . . . . . . . 5.00

790 Japonica flore pleno, $2-3 \mathrm{ft} . \ldots \ldots \ldots \ldots \ldots \ldots \ldots 12.00$

375 Japonica flore pleno, $18-24$ in. . . . . . . . . . 10.00

\section{MAHONIA}

100 Aquifolia, 18-24 in. $\quad \$ 20.00$

170 Aquifolia, $12-18$ in. ..................... 15.00

PHILADELPHUS

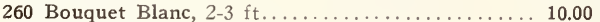

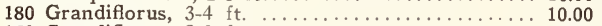

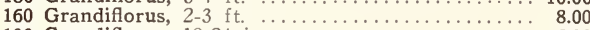

100 Grandiflorus, $18-24$ in. . . . . . . . . . . . . . . . 6.00

60 Lemoineii, 3-4 ft. . ...................... 10.00

500 Lemoineii, $2-3$ ft. . ......................... 8.00

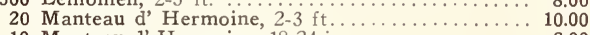

10 Manteau d' Hermoine, $18-24$ in.................... 8.00

250 Speciosa, 4-5 ft. . ..................... 12.00

435 Speciosa, 3-4 ft. . . . . . . . . . . . . . . . . . . . . . . . . 10.00

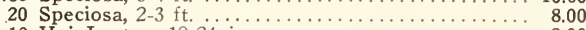

10 Voi Lactee, $18-24$ in. . . . . . . . . . . . . . . . . 8.00

PRIVET (Ligustrum)

3400 Amur River, $3-4 \mathrm{ft} . \ldots \ldots \ldots$ (Per 1000 . \$45) . \$ 5.00

900 Amur River, 2-3 ft. ............ (Per 1000. . 35) . 4.00

10000 California, 2-3 ft. .......... (Per 1000.. 25).. 3.00

8000 California, $18-24$ in. ........ (Per 1000 . 20) . 2.50

5000 California, $12-18$ in. .......... (Per 1000.. 15) .. 2.00

635 Regelianum, $24-30$ in. ..................... 8.00

270 Regelianum, $18-24$ in. .................. 6.00

PRUNUS

135 Regelianum, 12-18 in. ....................... 4.00

SPIREA

55 Aureum, 3-4 ft.

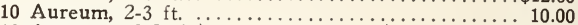

10 Aureum, $18-24$ in. ......................... 8.00

100 Aerifolia, $2-3 \mathrm{ft} . \ldots \ldots \ldots \ldots \ldots \ldots \ldots \ldots \ldots . \ldots 8.00$

35 Aerifolia, $18-24$ in. ............................. 6.00

50 Anthony Waterer, $8-12$ in. .................... 8.00

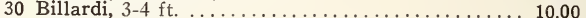

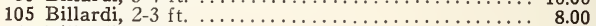

55 Billardi, $18-24$ in. ............................... 6.00

40 Billardi, $12-18$ in. ................................. 5.00

120 Callosa Alba, $12-18$ in. .................... 8.00

165 Opulifolia, 4-5 ft. ......................... 10.00

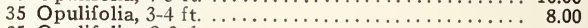

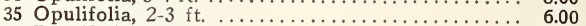

390 Prunifolia, $2-3 \mathrm{ft} . \ldots \ldots \ldots \ldots \ldots \ldots . . . . . .60$

210 Prunifolia, $18-24$ in. ........................ 8.00

215 Prunifolia, $12-18$ in. .......................... 6.00

5000 Van Houtte, $2-3 \mathrm{ft} \ldots \ldots \ldots \ldots \ldots$ (Per 1000.. $\$ 70) \ldots 8.6$

1000 Van Houtte, $18-24$ in.......... (Per 1000.. 50).. 6.00 SYMPHORICARPUS

125 Racemosus, $18-24$ in. ................... 88.00

445 Vulgaris, $2-3 \mathrm{ft} . \ldots \ldots \ldots \ldots \ldots \ldots \ldots \ldots \ldots . \ldots . \ldots . \ldots . \ldots$

85 Vulgaris, $18-24$ in. ..................... 6.00 TAMARIX

69 Vulgaris, $12-18$ in. .................... 5.00

750 Gallica, $4-5$ ft. . . . . . . . . . . . . . . . . . . $\$ 10.00$

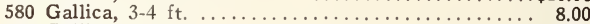

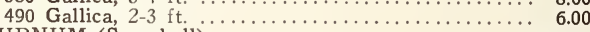
VIBURNUM (Snowball)

10 Lantana, 3-4 ft.

255 Opulus (High or Bush Cranberry), $2-3 \mathrm{ft} \ldots \ldots . .8 .00$

455 Opulus, (High or Bush Cranberry), 18-24 in.... 6.00

300 Opulus, (High or Bush Cranberry), 12-18 in. .... 5.00

500 Opulus Sterilis, (Common), 2-3 ft........... 10.00

2200 Opulus Sterilis, (Common), $18-24$ in. ............ 8.00

950 Plicatum, (Japan), 2-3 ft. . . . . . . . . . . . . . 15.00

1200 Plicatum, (Japan), $18-24$ in. ........................ 12.00

450 Plicatum, (Japan), 12-18 in. ............... 8.00

25 Tomentosum, 2-3 ft. .................... 12.00 WEIG0 Tome

60 Tomentosum, $18-24$ in. ...................... 10.00

90 Candida, $3-4$ ft. . . . . . . . . . . . . . . . . . . . $\$ 12.00$

500 Desboisii, 3-4 ft. .............................. 1200

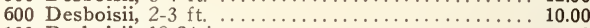

100 Desboisii, $18-24$ in. ......................... 8.00

345 Eva Rathke, $18-24$ in ........................ 15.00

100 Eva Rathke, 12-18 in ...................... 10.00

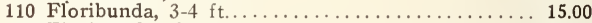

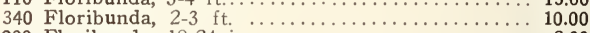

200 Floribunda, $18-24$ in ...................... 8.00

50 Mont Blanc, 3-4 ft. .................... 12.00

50 Mont Blanc, 2-3 ft. ................... 10.00

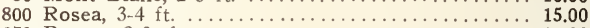

370 Rosea, 2-3 ft. ........................... 10.00

400 Rosea, $18-24$ in. .......................... 8.00

240 Variegata, $2-2 \mathrm{~T} / 2 \mathrm{ft} \ldots \ldots \ldots \ldots \ldots \ldots \ldots \ldots \ldots . . \ldots . \ldots . \ldots . . .60$

395 Variegata, $18-24$ in. .......................... 8.00

60 Typhina Laciniata, $4-5 \mathrm{ft} . \ldots \ldots \ldots \ldots \ldots \ldots \ldots . . . \ldots . . .60$

30 Typhina Laciniata, 3-4 ft. .............. 12.00

15 Typhina Laciniata, $2-3 \mathrm{ft}$.

8.00

\section{$L I L A C S$ \\ COMMON and PERSIAN}

COMMON PURPLE

34 Tree-form, $2-3 \mathrm{ft}$.

95 Bush-form, 18-24 in

245 Bush-form, 12-18 in

PERSIAN RED

20 Bush-form, 3-4 ft. .

355 Bush-form, 2-3 ft.
Per 100

$\$ 15.00$

8.00

6.00

15.00

12.00

\section{COMMON WHITE}

Per 100

15 Tree-form, $3-4$ ft. $\ldots \ldots \ldots \ldots \ldots \ldots \ldots \ldots \ldots \ldots . \ldots \ldots$

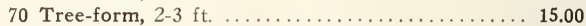

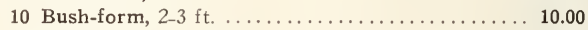

70 Bush-form, $18-24$ in. . . . . . . . . . 6.00

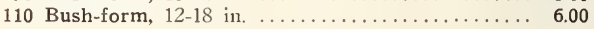

\section{LILACS-Named Varieties}

Tree-form, 2-3 ft.

$\$ 25.00$

Bush-form, 3-4 ft. 
$\mathrm{S}$, single; $\mathrm{D}$, double.

Alphonse Lavallee. D. Blue shading to violet; extra large panicles

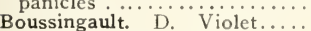

$\begin{array}{llll}12 & \ldots & \ldots & 10\end{array}$

Congo. S. Wine-red . Preitschneider. D. Purplish

Dr. Breitschneider. D. Purplish
in bud, opening white. Late...

Dr. Masters. D. Clear lilac, with

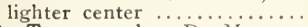

Dr. Troyanowsky. D. Mauve...

Frau Dammann. S. Pure white

Japonica. S. Creamy-white. Late

Josikaea. S. Purple. Late....... 125145

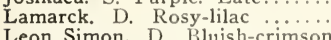

Leon Simon. D. Bluish-crimson

Marc Michaeli. D. Clear lilac-blue

\section{LILACS-NAMED VARIETIES-Continued}

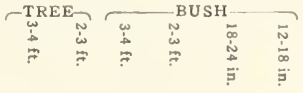

Marie Legraye. S. Pure white. .

Mme. Lemoine. D. Pure white.

Michael Buchner. D. Dwarf. Lilac

Othello. S. Dark, purplish-red.

President Viger. D. Bluish-lilac Princess Alexandra. S. White.

Rubra de Marley. S. Red.

Souvenir de Ludwig Spaeth. S. Purplish red

Toussaint L'Ouverture. S. Dark purplish flowers ................

Villosa. S. Light purple in bud opening white. Late

Vulcan. S. Ruby-red.

William Robinson. S. Violetmauve .......... S. Violet-

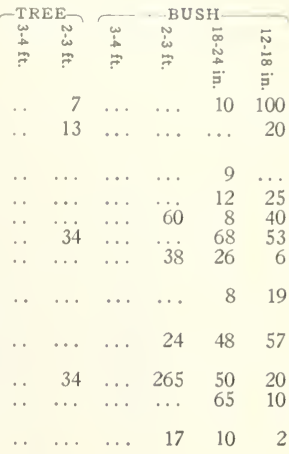

\section{ORNAMENTAL and SHADE TREES}

BEECH $\begin{array}{r}\text { Per } 100 \\ \text { Per } 100\end{array}$

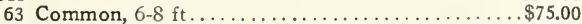

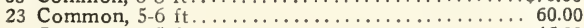

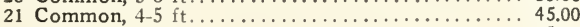

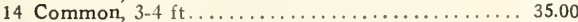

50 Common, 2-3 ft............................... 2500

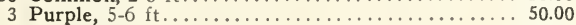

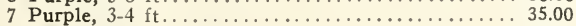

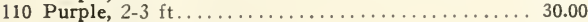

$\mathrm{BIRCH}$

50 Purple, $18-24$ in...................................... 25.00

200 Cut-leaved Weeping, 6-8 ft............... 50.00

195 Cut-leaved Weeping, 5-6 ft................. 35.00

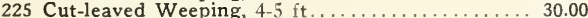

245 Cut-leaved Weeping, $3-4 \mathrm{ft} \ldots \ldots \ldots \ldots \ldots \ldots \ldots \ldots \ldots \ldots \ldots \ldots \ldots .25 .00$

CATALPA

600 Bungeii, 1 yr.......................... 40.00

CHERRY

80 Japan Weeping, pink, 2 yr. heads............ 75.00

CHESTNUT

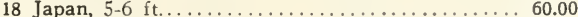

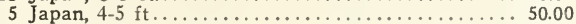

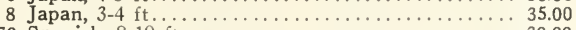

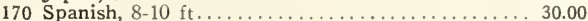

500 Spanish, 6-8 ft ......................... 25.00

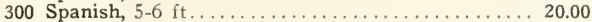

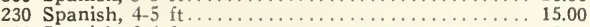

20 Spanish, 3-4 ft........................ 12.00

CORNUS

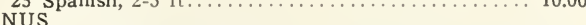

380 Florida Rubra, $2-2 \frac{1}{2} \mathrm{ft} \ldots \ldots \ldots \ldots \ldots \ldots \ldots \ldots . \ldots \ldots$

CRAB, Double Flowering

140 Bechtel's, 2-3 ft.......................... 25.00

130 Bechtel's, $18-24$ in ...................... 20.00

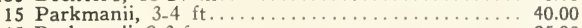

ELM

45 Parkmanii, 2-3 ft.............................. 25.00

48 American, $10-12 \mathrm{ft}$. $(11 / 2-13 / 4$ in. $) \ldots \ldots \ldots \ldots \ldots . \ldots 50.00$

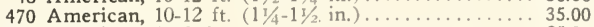

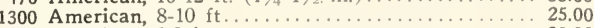

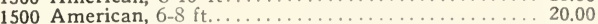

HORSE CHESTNUT

12 Horse Chestnut, $8-10 \mathrm{ft} \ldots \ldots \ldots \ldots \ldots \ldots \ldots . \ldots \ldots . \ldots \ldots$

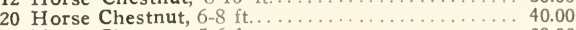

15 Horse Chestnut, $5-6 \mathrm{ft} \ldots \ldots \ldots \ldots \ldots \ldots \ldots \ldots \ldots \ldots . \ldots \ldots . \ldots \ldots$

LINDEN

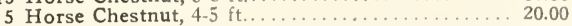

14 American, $10-12 \mathrm{ft}$. .

90 American, 8-10 ft.

50.00

40.00

30.00

90 American, 6-8 ft.

7 European, $12 \mathrm{ft}$. ( $13 / 4 \mathrm{in}$.

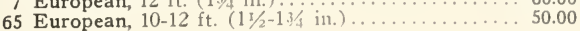

LINDEN

Per 100

11 European, $10-12 \mathrm{ft} .(13 / 4-1 \mathrm{r} / 2$ in.) ............ $\$ 40.00$

235 European, 8-10 ft........................... 35.00

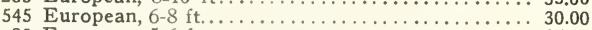

MAGNO Euro

OLIA 4 Ko 500

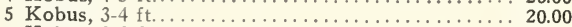

MAPLE

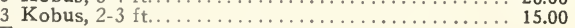

For Maple in sizes suitable to line out, see page 15.

12 Norway, $12-14 \mathrm{ft} .(2-3$ in.) ................... 80.00

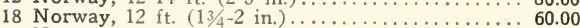

90 Norway, $10-12 \mathrm{ft} .\left(1 \frac{1}{2}-13 / 4 \mathrm{in}\right) \ldots \ldots \ldots \ldots \ldots \ldots . . .50 .00$

160 Norway, $10-12 \mathrm{ft}$. (11/4-11/2 in.) ................ 40.00

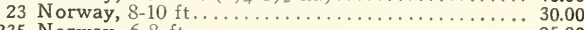

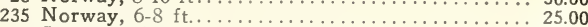

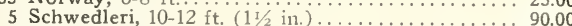

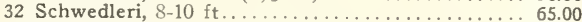

20 Schwedleri, 6-8 ft..................... 50.00

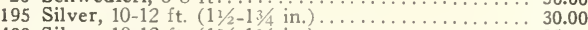

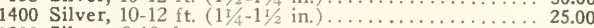

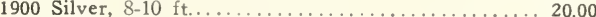

500 Silver, 6-8 ft........................ 15.00

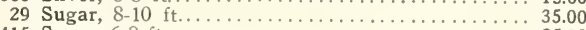

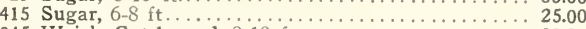

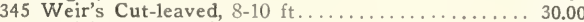

210 Weir's Cut-leaved, 6-8 ft.......... 20.00

MULBERRY

80 Tea's Weeping, 2 yr........................ 60.00

POPLAR

75 Lombardy, $12-14$ ft. (2-2 $1 / 1 \mathrm{in}$.$) .............. 50.00$

145 Lombardy, $12-14$ ft. $(13 / 4-2$ in. $) \ldots \ldots \ldots \ldots \ldots . . . . . .400$

315 Lombardy, $10-12 \mathrm{ft} .(11 / 2-134$ in. $) \ldots \ldots \ldots \ldots \ldots . \ldots . . .60$

50 Lombardy, $10-12 \mathrm{ft}$ (11/1-11/2 ill) ........ 25.00

PLANE

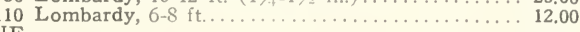

THORN

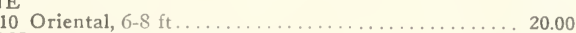

4 Azarolus Carrieri, $8-10 \mathrm{ft} \ldots \ldots \ldots \ldots \ldots \ldots \ldots \ldots . \ldots . \ldots . \ldots 00$

10 Azarolus Carrieri, $6-8 \mathrm{ft} \ldots \ldots \ldots \ldots \ldots \ldots \ldots \ldots . \ldots \ldots . \ldots \ldots$

25.00

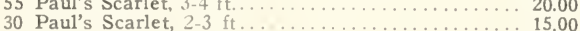

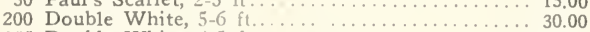

155 Double White, $4-5 \mathrm{ft} \ldots \ldots \ldots \ldots \ldots \ldots \ldots \ldots . \ldots 25.00$

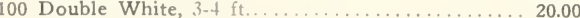

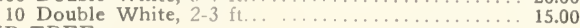

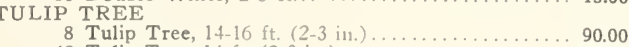

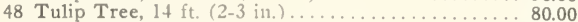

10 Tulip Tree, $12-14 \mathrm{ft} .\left(11^{3}-2 \mathrm{in}.\right) \ldots \ldots \ldots \ldots \ldots .60 .00$

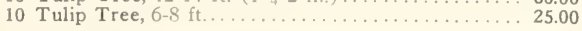

We cannot undertake to supply long and much assorted lists of stock calling for only small quantities of each kind, save at an advance of 10 to 20 per cent. above our printed rates. 


\section{FRUIT DEPARTMENT}

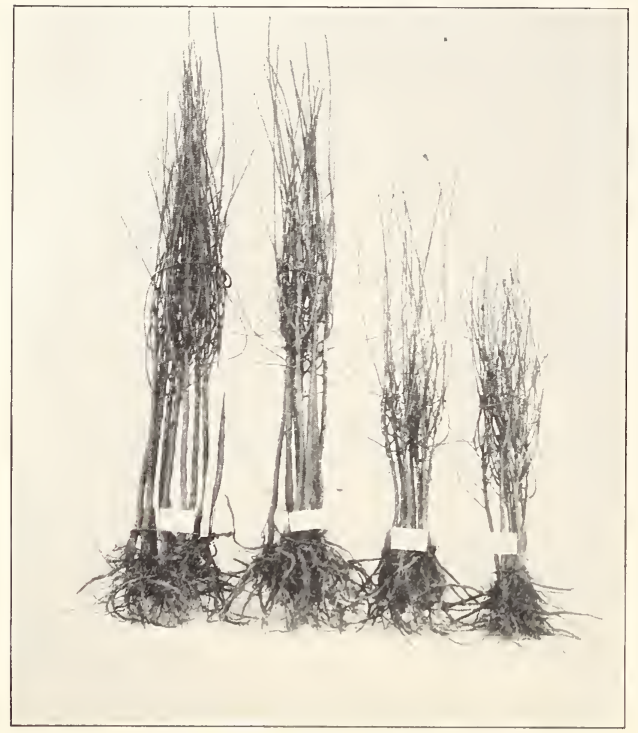

Apples, Standard and Dwarf

\section{APPLES, Standard, 2 years}

\begin{tabular}{|c|c|c|c|c|c|}
\hline & $1 \mathrm{in.}$ & 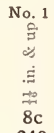 & 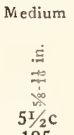 & 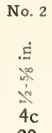 & 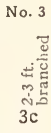 \\
\hline Alexander $\ldots \ldots \ldots \ldots \ldots$ & $\cdots$ & 240 & 195 & 20 & \\
\hline $\begin{array}{l}\text { Arkansas Black } \ldots \ldots \ldots \ldots \ldots \\
\text { Baldwin } . . \ldots \ldots \ldots \ldots\end{array}$ & 300 & $\begin{array}{r}20 \\
3000\end{array}$ & $\begin{array}{r}7 \\
855\end{array}$ & $\ddot{895}$ & 190 \\
\hline Baxter ............. & $\cdots$ & $\cdots$ & $\because$ & 25 & \\
\hline Belleflower . . ......... & $\cdots$ & 45 & 10 & & \\
\hline Ben Davis $\ldots \ldots \ldots \ldots \ldots \ldots$ & $\cdots$ & 210 & 99 & 30 & \\
\hline Caroline Red June........ & $\cdots$ & 10 & 8 & 10 & \\
\hline Delicious ${ }^{\prime} \ldots \ldots \ldots \ldots$ & $\cdots$ & 780 & 479 & 440 & 00 \\
\hline Duchess of Oldenburg..... & $\cdots$ & 2700 & 785 & 600 & \\
\hline Early Harvest $\ldots \ldots \ldots \ldots \ldots$ & $\cdots$ & $\ddot{i i}$ & 6 & 25 & \\
\hline Fall Pippin $\ldots \ldots \ldots \ldots \ldots \ldots$ & $\cdots$ & 115 & 19 & 110 & \\
\hline Fameuse $\ldots \ldots \ldots \ldots \ldots \ldots$ & $\cdots$ & 1230 & 445 & 675 & \\
\hline 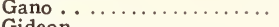 & $\cdots$ & 345 & 140 & 90 & \\
\hline 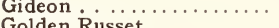 & $\cdots$ & 9 & $\cdots$ & & \\
\hline $\begin{array}{l}\text { Golden Russet } \ldots \ldots \ldots \ldots \ldots \\
\text { Gravenstein . . . . . . . } \ldots \ldots \ldots\end{array}$ & $\cdots$ & & 7 & 5 & \\
\hline $\begin{array}{l}\text { Gravenstein } \ldots \ldots \ldots \ldots \ldots \\
\text { Grimes Golden } \ldots \ldots \ldots \ldots \ldots\end{array}$ & $\cdots$ & 100 & iiv & & \\
\hline $\begin{array}{l}\text { Grimes Golden } \ldots \ldots \ldots \ldots \ldots \\
\text { Hyslop } \ldots \ldots \ldots \ldots \ldots \ldots\end{array}$ & $\cdots$ & 46 & 112 & 14 & \\
\hline Hubbardston's Nonesuch & $\cdots$ & $\begin{array}{l}150 \\
370\end{array}$ & $\begin{array}{r}33 \\
185\end{array}$ & 155 & \\
\hline Jonathan ............ & $\cdots$ & $\ldots$ & $\begin{array}{r}103 \\
63\end{array}$ & 100 & \\
\hline King ${ }^{\prime} \ldots \ldots \ldots \ldots \ldots \ldots$ & 50 & 875 & 204 & 200 & \\
\hline Longfield . . . . . . . . . & $\ldots$ & 86 & 18 & 15 & \\
\hline McIntosh Red .......... & $\cdots$ & 4780 & 1425 & 794 & 225 \\
\hline Maiden Blush $\ldots \ldots \ldots \ldots \ldots$ & $\cdots$ & 69 & & 20 & \\
\hline Mann $\ldots \ldots \ldots \ldots \ldots \ldots$ & $\cdots$ & 62 & 15 & & \\
\hline N. W. Greening ............ & $\cdots$ & 840 & 144 & 82 & 2 \\
\hline Mammoth Black Twig..... & $\ldots$ & 116 & 16 & 20 & \\
\hline Northern Spy $\ldots \ldots \ldots \ldots \ldots$ & $\cdots$ & 2145 & 433 & 610 & 125 \\
\hline Pewaukee . . . . . . . . . . & $\cdots$ & $\ddot{B}$ & 40 & & \\
\hline Rambo . . . . . . . . . . & $\cdots$ & 95 & 33 & 10 & \\
\hline Red Astrachan $\ldots \ldots \ldots \ldots$ & $\ldots$ & 200 & 135 & 180 & \\
\hline
\end{tabular}

APPLES, Standard, 2 years-Continued

\begin{tabular}{|c|c|c|c|c|c|}
\hline & $1 \mathrm{in.}$ & No. 1 & Medium & No. 2 & No. 3 \\
\hline & & $\ddot{z}$ &.$\Xi$ &.$\dot{g}$ & ש \\
\hline & & $\dot{\Xi}$ & $\nRightarrow$ & $\infty$ & \\
\hline & & to & $\infty$ & & \\
\hline & $10 \mathrm{c}$ & $8 \mathrm{c}$ & $5^{1} / 2 c$ & $4 c$ & \\
\hline R. I. Greening. . & $\ldots$ & 700 & 80 & 20 & \\
\hline Rome Beauty ... & $\cdots$ & 115 & 168 & 100 & \\
\hline Roxbury Russet . & $\ldots$ & & 7 & 7 & \\
\hline Scott Winter .... & $\ldots$ & 23 & 20 & 30 & \\
\hline Seek-no-Further . & $\ldots$ & & 62 & & \\
\hline Smokehouse . . ... & $\ldots$ & 70 & 3 & 15 & \\
\hline Spitzenburg. & $\ldots$ & & 210 & 60 & \\
\hline Stark . . . . . & $\ldots$ & 925 & 10 & 13 & \\
\hline man's Winesap ... & $\ldots$ & 90 & 63 & 50 & \\
\hline Sweet Bough ............... & $\ldots$ & 105 & 19 & 10 & \\
\hline Taln & $\ldots$ & 90 & 19 & 9 & \\
\hline Transcendent ... & $\ldots$ & 110 & 10 & & \\
\hline y Ounce $\ldots \ldots \ldots \ldots$ & & & 11 & 27 & \\
\hline er . . ........... & $\ldots$ & 1910 & 280 & 100 & \\
\hline$\therefore \ldots \ldots \ldots \ldots \ldots$ & $\cdots$ & 1460 & 588 & 310 & \\
\hline Whitney's Seedling ........ & $\ldots$ & 59 & 6 & 28 & \\
\hline Winesap ............... & $\ldots$ & 25 & & & \\
\hline Winter Banana ......... & $\ldots$ & 210 & 20 & 30 & \\
\hline f River .... & $\ldots$ & 1670 & 290 & 275 & \\
\hline Yellow Transparent ....... & $\cdots$ & & 64 & 220 & \\
\hline ork Imperial .......... & $\ldots$ & 90 & 28 & 75 & \\
\hline
\end{tabular}

\section{APPLES, Dwarf, 2 years}

\begin{tabular}{|c|c|c|c|c|}
\hline & $\begin{array}{c}\text { No. } 1 \\
5 / 8 \text { in. \& up } \\
25 \mathrm{c}\end{array}$ & $\begin{array}{l}\text { Medium } \\
1 / 2-5 / 8 \text { in. } \\
20 \mathrm{c}\end{array}$ & $\begin{array}{l}\text { No } 2 \\
3 / 8 \text { in. } \\
15 \mathrm{c}\end{array}$ & $2-3 \mathrm{ft}$. \\
\hline \multicolumn{5}{|l|}{ Baldwin } \\
\hline Duchess of Oldenburg & $\ldots 340$ & 350 & 125 & \\
\hline Fameuse... & 325 & 125 & & \\
\hline Gravenstein . & & & & \\
\hline King . . . ...... & 166 & 57 & & \\
\hline McIntosh Red & $\begin{array}{l}100 \\
160\end{array}$ & 510 & 160 & \\
\hline Northern Spy & 339 & 418 & 228 & \\
\hline Red Astrachan ..... & & 90 & & \\
\hline R. I. Greening. . ....... & $\begin{array}{ll}\ldots & 94\end{array}$ & 5 & $\cdots$ & \\
\hline ome Beauty ....... & & 53 & $\ldots$ & \\
\hline nan's Winesap & $\ldots \quad 154$ & & & \\
\hline$\therefore \ldots \ldots$ & ... 70 & 112 & $\ldots$ & \\
\hline $\mathrm{y} \ldots \ldots \ldots$ & 17 & & & \\
\hline Yellow Transparent & 96 & 102 & $\ldots$ & \\
\hline
\end{tabular}

CANADA, November 2, 1914.- "I thank you for the extra size of many plants. The Crimson Ramblers secm to have made great growth this season."

ILLINOIS, November 5, 1914.--"Clematis, $\mathrm{Hy}$ drangeas, Roses; this stock was fine. Among the best we ever had."

IOWA, November 13, 1914.- "Enclosed find check for Roses. Will want more in Spring. They certainly cannot be beat."

INDIANA, October 22, 1914.- - Vines and Perennials. "Goods very satisfactory and in fine condition. The kind of stock we want. Will buy more."

This list is intended for the trade only, and the prices given will be applied only when the quantities required are such as to entitle the purchaser to wholesale rates. 


\section{FRUIT DEPARTMENT-Continued}

CHERRIES, on Mahaleb, 2 years

Early Richmond $\begin{array}{ccc}\text { No. } 1 & \text { Medium } & \text { No. } 2 \\ 3 / 4 \text { in. \& up } & 5 / 8-3 / 4 \text { in. } 5 / 2-5 / 8 \text { in. }\end{array}$

English Morello $20 \mathrm{c} \quad 15 \mathrm{c}$

Montmorency.

830

190

\section{CHERRIES, on Mazzard, 2 years}

IVe especially recommend Cherries grown on Mazzard stocks. They are nuch longer lived and more vigorous than on Mahaleb. They are the only trees for orchard planting. That is why the trees cost more-and they are worth more.

\begin{tabular}{|c|c|c|c|}
\hline & $\begin{array}{c}\text { No. 1 } \\
3 / 4 \text { in. \& up } \\
25 \mathrm{c}\end{array}$ & $\begin{array}{l}\text { Medium } \\
5 / 8-3 / 4 \text { in. } \\
18 \mathrm{c}\end{array}$ & $\begin{array}{l}\text { No. } 2 \\
1 / 2-5 / 8 \text { in. } \\
12 \mathrm{c}\end{array}$ \\
\hline $\begin{array}{l}\text { Black Tartarian } \\
\text { Early Richmond .. }\end{array}$ & $\ldots \ldots \ldots \ldots \ldots{ }_{44}$ & 38 & \\
\hline $\begin{array}{l}\text { Early Kichmond .. } \\
\text { English Morello .. }\end{array}$ & ${ } \ldots \ldots \ldots \ldots \ldots \ldots{ }^{49}$ & 110 & 12 \\
\hline Governor Wood .. & & $\ldots$ & . \\
\hline $\begin{array}{l}\text { Montmorency ..... } \\
\text { Rockport Bigarrea }\end{array}$ & $\ldots \ldots \ldots \ldots \ldots \ldots 85$ & $\cdots$ & \\
\hline $\begin{array}{l}\text { Rockport Bigarrea } \\
\text { Windsor }\end{array}$ & au $\ldots \ldots \ldots \ldots \ldots \ldots$ & $\cdots$ & 15 \\
\hline Yellow Spanish & $\ldots \ldots \ldots \ldots \ldots 210$ & $\cdots$ & $\ldots$ \\
\hline
\end{tabular}

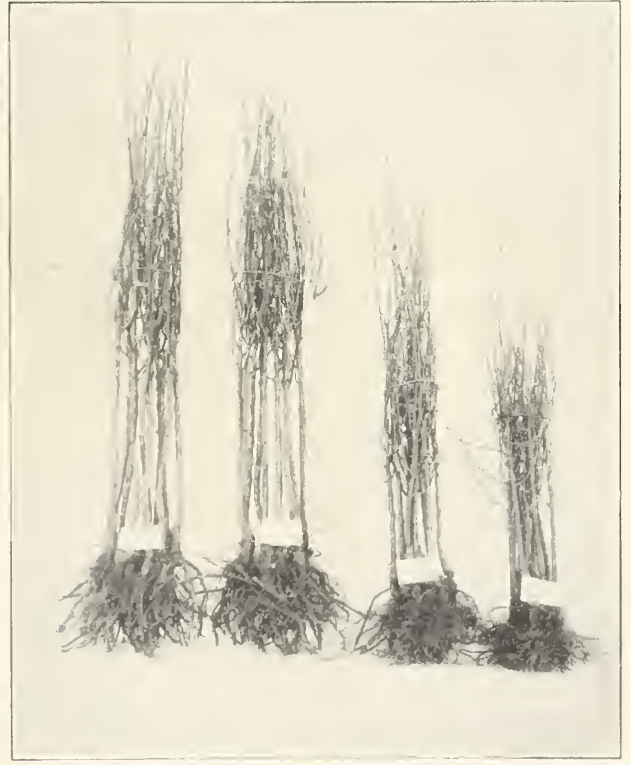

Pears, Standard and Dwarf

\section{PEARS, Standard, 2 Years}

\begin{tabular}{|c|c|c|c|c|c|}
\hline & $1 \mathrm{in.}$ & $\begin{array}{l}\text { No. } 1 \\
\text { th in. \& up } \\
12 \mathrm{c}\end{array}$ & $\begin{array}{c}\text { Medium } \\
58 .+2 \text { in. } \\
9 c\end{array}$ & $\begin{array}{l}\text { No. } 2 \\
\pi / 2 \cdot 5 / 8 \text { in. } \\
6 \mathrm{c}\end{array}$ & $\begin{array}{r}2-3 \mathrm{ft} . \\
4 \mathrm{C}\end{array}$ \\
\hline Bartlett .......... & ... & 2800 & 440 & 775 & 165 \\
\hline Beurre d'Anjou ..... & $\ldots$ & 520 & 97 & 120 & 28 \\
\hline Beurre Clairgeau & $\ldots$ & 글 & ‥ & 16 & \\
\hline Clapp's Favorite ...... & $\ldots$ & 2700 & 189 & 250 & 60 \\
\hline Duchess d'Angouleme .... & $\cdots$ & 369 & 50 & 28 & 10 \\
\hline Flemish Beauty ........ & $\ldots$ & $\ldots$ & $\ldots$ & 23 & 10 \\
\hline
\end{tabular}

PEARS, Standard, 2 Years-Continued

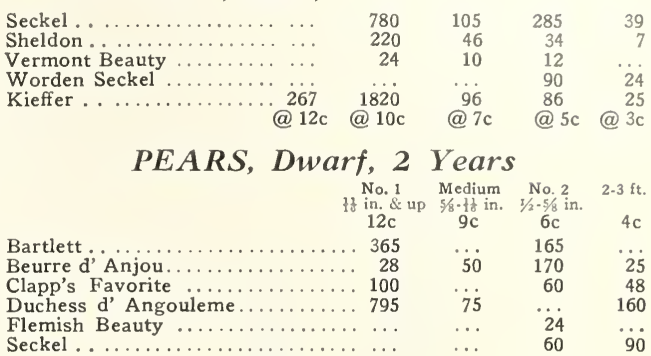

\section{PEACHES, 1 Year}

\begin{tabular}{|c|c|c|c|c|}
\hline & $\begin{array}{l}\text { No. } 1 \\
\text { io in. \& up } \\
6 \mathrm{c}\end{array}$ & $\begin{array}{c}\text { Medium } \\
\frac{7}{18}-P^{2} \mathrm{in} . \\
4 \mathrm{C}\end{array}$ & $\begin{array}{c}\text { No. } 2 \\
f^{10}-\frac{7}{16} \text { in. } \\
3 \mathrm{c}\end{array}$ & $\begin{array}{r}2-3 \mathrm{ft} . \\
2 \mathrm{c}\end{array}$ \\
\hline Admiral Dewey .............. & . . 105 & 10 & 11 & \\
\hline 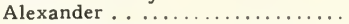 & .. 235 & 45 & $\ldots$ & \\
\hline Beers' Smock ................. & . . ... & $\ldots$ & 140 & \\
\hline 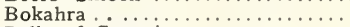 & $\ldots \ldots$ & $\ldots$ & 20 & \\
\hline Belle of Georgia................ & .. 69 & $\ldots$ & $\ldots$ & \\
\hline 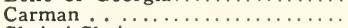 & $\therefore 840$ & $\ldots$ & 6 & \\
\hline 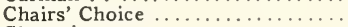 & $\ldots \ldots$ & $\ldots$ & 18 & \\
\hline 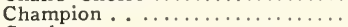 & $\ldots 10$ & $\ldots$ & $\ldots$ & \\
\hline Crosby . . . . . . . & $\therefore \ldots$ & & 17 & \\
\hline 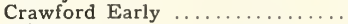 & .. 495 & 290 & 167 & \\
\hline Elberta . . . . . . . . . . . & .. 189 & 495 & 225 & \\
\hline 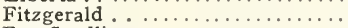 & $\ldots \ldots$ & 215 & 39 & \\
\hline 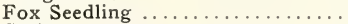 & . 429 & 115 & $\ldots$ & \\
\hline Globe . . . . . . . . . . . . & . 19 & $\ldots$ & $\ldots$ & \\
\hline 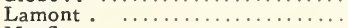 & $\ldots 1000$ & 300 & 64 & \\
\hline Mayflower . . . . . . . . . & . 149 & 12 & 18 & \\
\hline 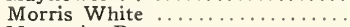 & $\therefore 77$ & 155 & & \\
\hline 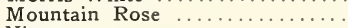 & $\ldots \ldots$ & 38 & 25 & \\
\hline 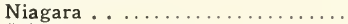 & .. 130 & 60 & & \\
\hline 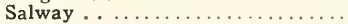 & $\ldots \ldots$ & 30 & 28 & \\
\hline mp-the-World . . . . . . . . . & $\ldots \ldots$ & 11 & 45 & \\
\hline umph $\ldots \ldots \ldots \ldots \ldots \ldots \ldots$ & .. 20 & $=$ & 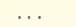 & \\
\hline
\end{tabular}

NEW YORK, Deeember 8, 1914.- "Right here we wish to thank you for the exeellent quality of the goods supplied us and for your prompt and eourteous attention."

OHIO, September 28, 1914.- “Arrived promptly and in good condition."

PENNSYLVANIA, September 29, 1914.- "Plants fine! And in perfeet eondition."

IILINOIS, Otcober 6, 1911.- "Arrived in very excellent condition."

IOWA, October 31, 191.-- "The Roses are fine. splendid roots!"

CONNECICUT, November 17, 1911.- "Came in fine shape. Send me the same next year."

We cannot undertake to supply long and much assorted lists of stock calling for only small quantities of each kind, save at an advance of 10 to 20 per cent. above our printed rates. 


\section{FRUIT DEPARTMENT-Continued}

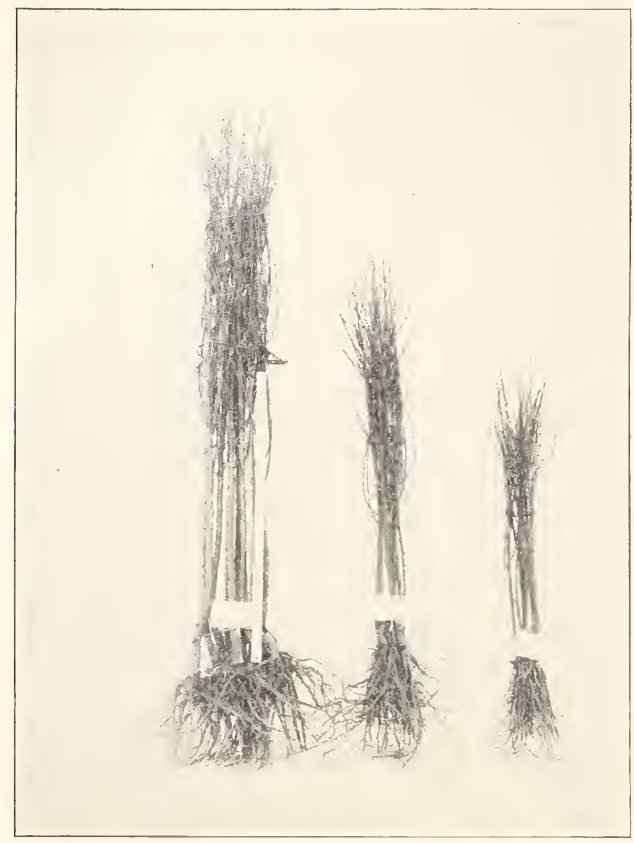

Peaches, Three Grades

PLUMS, 2 Years

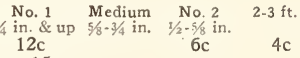

Abundance . . 15

Bradshaw 50

Burbank .....

German Prune

....... 250

Lombard . . .

475

Monarch .

Reine Claude

Shipper's Pride

Shropshire Damson

Tennant Prune

York State Prune

QUINCES, 2 years

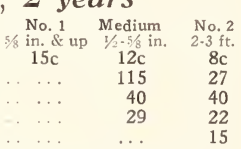

Bentley...

Meech's Prolific

Rea's Mammoth

\section{SMALL FRUITS}

\section{CURRANTS, 2 years}

No. 1
Per 1000
$750 @ \$ 30$
$5000 @ 25$
$5500 @ 75$
$5000 @ 25$
Medium
Per 1000

Black Victoria

Fay's Prolific

Perfection .

White Grape
GOOSEBERRIES, 2 years

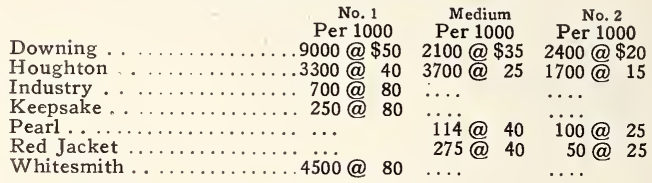

CONNECTICUT, November 12, 1914. - "The plants are fine and in excellent eondition."

INDIANA, October 25, 1914.- "Roses arrived in perfeet condition and very fine stoek."

PENNSYLVANIA, November 4,1914 .- "Ail good clean stock."

IVISCONSIN, November 28, 1914.- "Roses arrived in good order."

NORTH DAKOTA, November 29, 191\%.-“Good condition--good stock."

ILI.INOIS, Norember 25, 1914.--"Roses arrived in good condition."

OHIO, November 25, 1914.- "Roses arrived in good condition."

OHIO. Norember 18, 1911.- "Roses arrived in good conditien."

MISSOURI, November 30, 1914.--"Roses in good condition."

MICHIGAN, December 2, 1914.- "Shrubs and Roses received in good shape. Thanks for same."

ILLINOIS, November 21, 1914.-"Roses arrived in finc condition."

PENNSYLVANIA, November.5, 1914.--"Received your shipment of Hydrangea and Roses. They are an cxecedingly fine lot."

This list is intended for the trade only, and the prices given will be applied only when the quantities required are such as to entitle the purchaser to wholesale rates. 


\section{FOR LINING OUT}

Maples, Norway, 7 to $8 \mathrm{ft}$., $\$ 70$ per 1000 . These Maples are imported, three years old, twice-transplanted, slender, but straight as a ramrod. If you start rightwith a straight stem, time will add the head and the caliper; but a crooked tree is always going to remain a crooked tree.

Maples, Norway, 5 to $7 \mathrm{ft}$., . . $\$ 60$ per 1000 Maples, Sugar, 21/2 to $3 \mathrm{ft}$, home-

Maples, Norway, 4 to $5 \mathrm{ft.}$. . $\$ 50$ per $1000 \quad$ grown, not collected, . . $\$ 60$ per 1000

Maples, Sugar, $3 \frac{1}{2} 2$ to $5 \mathrm{ft}$., . . $\$ 90$ per 1000 Maples, Sugar, 18 to 24 in., $\quad$. $\$ 30$ per 1000

Nursery-grown, transplanted, imported. $\quad$ Maples, Sugar, 10 to 18 in., $\quad \$ \quad \$ 15$ per 1000

Manetti Rose Stocks, English-grown, re-graded, well-rooted, 3 to $5 \mathrm{~m} / \mathrm{m}, \$ 9.50$ per $1000 ; 5$ to $9 \mathrm{~m} / \mathrm{m}, \$ 11.00$ per 1000 .

Apple Seedlings, French, heaviest grade, 7 to $12 \mathrm{~m} / \mathrm{m}, \$ 9.00$ per 1000 .

Cherry, Mahaleb, French, 5 to $9 \mathrm{~m} / \mathrm{m}, \$ 6.50$ per 1000 .

Duty paid.

Apple Seedlings, Kansas-grown :

No. 1 Straight, 3-16 in. and up, $\$ 6.50$ per 1000 "Grafters,"

$\$ 2.50$ per 1000

No. 2 Straight, 2-16 to 3-16 in, $\$ 3.00$ per 1000 No. 3, under 2-16 in., . $\$ 1.25$ per 1000

f. o. b. here, packing at cost.

FRENCH STOCKS. We sell these, both the Fruit Tree Seedlings and Ornamentals of all kinds in sizes suitable for nursery planting, for Mr. Charles Detriche, Sr., Angers, France, whose new complete catalog will be sent postfree on application.

\section{Surplus Apple Scions $\$ 1.25$ per 1000}

Varieties: Duchess, Stayman, Grimes Golden, Baldwin, Hubbardston, Bismarck, Stark, N. W. Greening, Wagener, Rome Beauty, Northern Spy, Talman Sweet, Red Astrachan, Wealthy, Winter Banana, Fameuse, Wolf River, Delicious, McIntosh.

HYDRANGEA OTAKSA. Fine plants for blooming in pots to sell Decoration Day. These are pot-grown out-doors all summer; strong branches; good flower buds; with 7 to 10 branches, $\$ 25$ the hundred; 5 and 6 branches, $\$ 20$ the hundred; 4 branches, $\$ 15$ the hundred. The newest and best French varieties, $\$ 25$ the hundred-all in 6-inch pots.

RHODODENDRONS. We have imported $10,(000$ plants annually for the past four years. We offer another fine lot of 18 to 24 -inch plants at $\$ 60$ the hundred.

We cannot undertake to supply long and much assorted lists of stock calling for only small quantities of each kind, save at an advance of 10 to 20 per cen $:$. above our printed rates. 


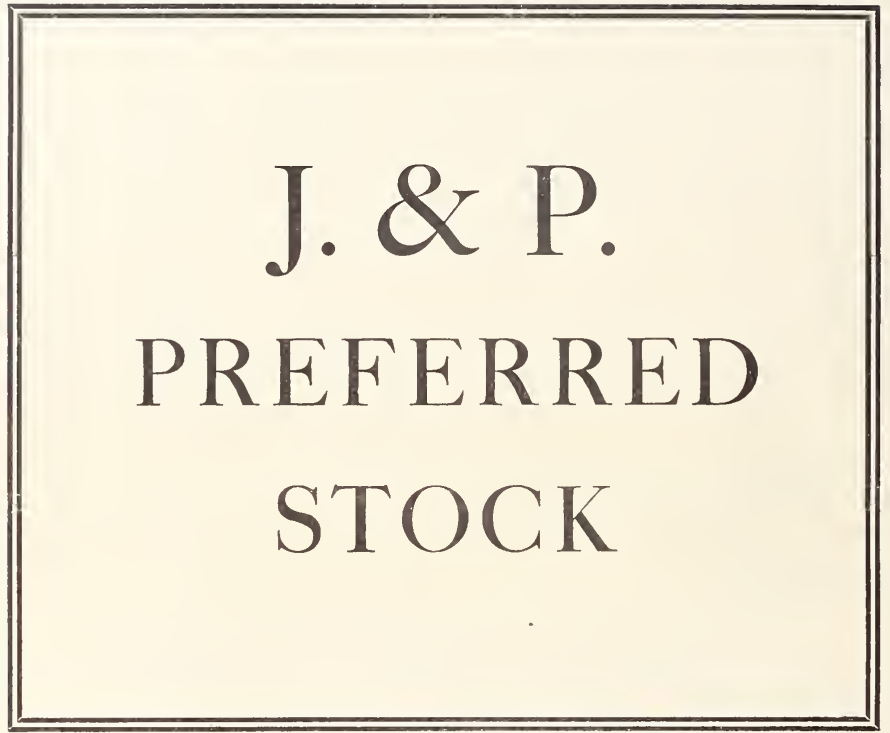

THE DUBOIS PRESS Rochester, N.Y. 